\title{
Supplementary
}

\section{Structure-Based Rational Design of Two Enhanced Bacterial Lipocalin Blc Tags for Protein-PAINT Superresolution Microscopy}

Liya Muslinkina ${ }^{\star} \|,{ }^{*}$, Alexey S. Gavrikov ${ }^{*}{ }^{*}$, Nina G. Bozhanova ${ }^{\S}$, Alexander S. Mishin *,

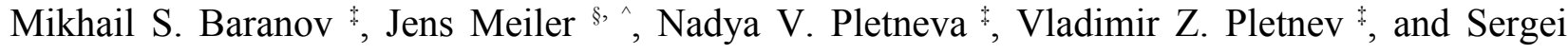
Pletnev ${ }^{\dagger}, * *$

${ }^{\dagger}$ Basic Research Program, Frederick National Laboratory for Cancer Research, Argonne, IL 60439, USA

\$ Shemyakin-Ovchinnikov Institute of Bioorganic Chemistry, Russian Academy of Sciences, Moscow 117997, Russian Federation

$\S$ Department of Chemistry, Center for Structural Biology, Vanderbilt University, Nashville, TN 37235, USA

Institute for Drug Discovery, Leipzig University, Leipzig, SAC 04103, Germany

*Both authors contributed equally to this manuscript.

${ }^{* *}$ Corresponding author email: pletnevs@anl.gov

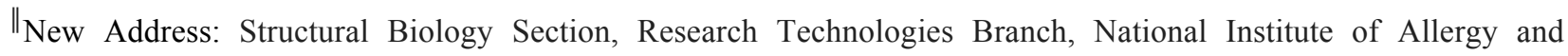
Infectious Diseases, National Institutes of Health, Bethesda, Maryland 20852, USA

Supplementary Table $\quad 2$

Supplementary Figures $\quad 3$

$\begin{array}{ll}\text { Supplementary Movies } & 6\end{array}$

$\begin{array}{ll}\text { Materials and Methods } & 7\end{array}$

Supplementary References 11 
Table 1. Crystallographic data and refinement statistics

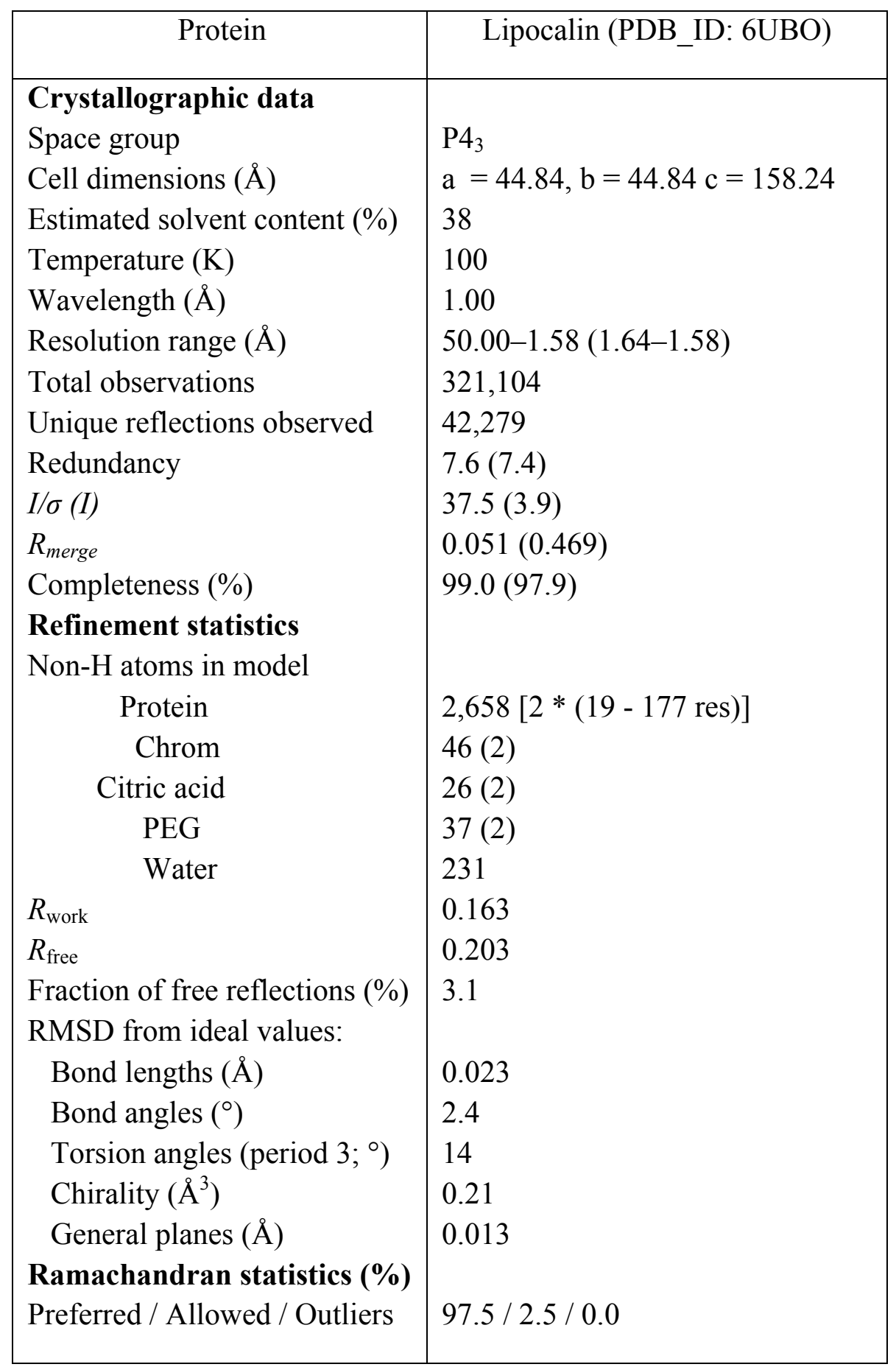

Values in parentheses are given for the highest-resolution shells. 

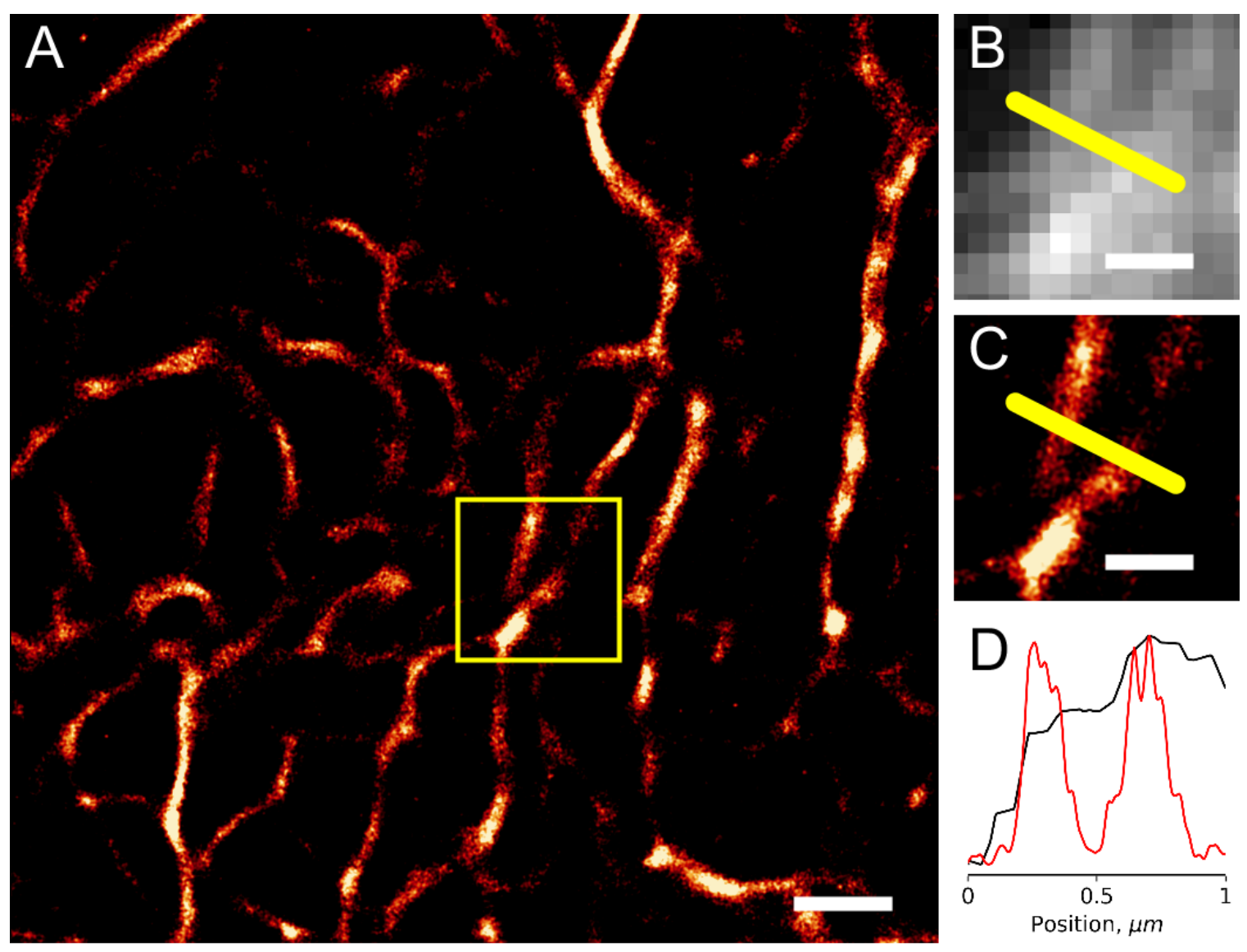

Figure S1. Super-resolution microscopy with DiB3/F74V. (A-D) Super-resolution imaging of HeLa cells transiently transfected with vimentin- DiB3/F74V in presence of $15 \mathrm{nM} \mathrm{M739}$. Widefield image (B) and super-resolution reconstruction (C) from 5,000 frames are shown; scale bars are $0.5 \mu \mathrm{m}$. (D) Normalized intensity profiles of yellow lines shown on the widefield and super-resolution images (B and C); black curve - widefield and red curve - super-resolution. 

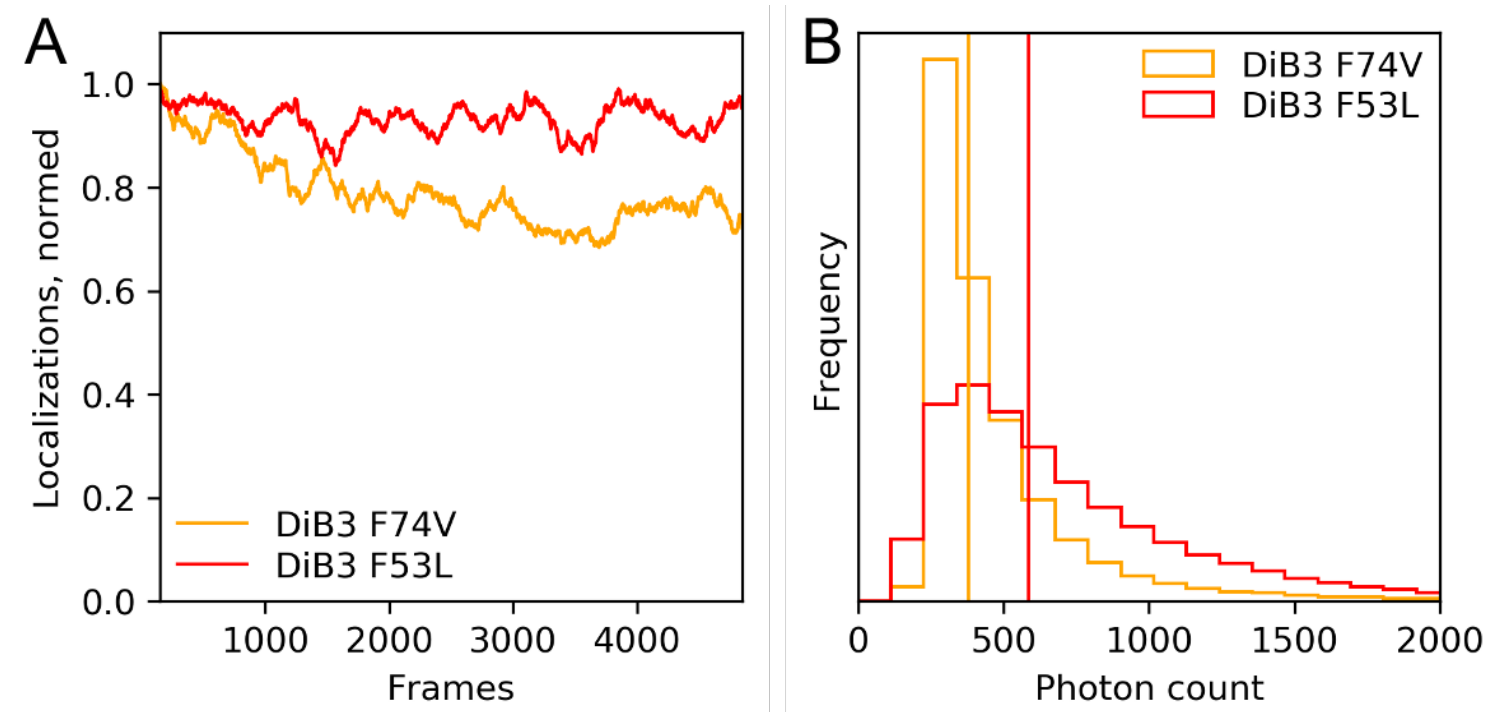

Figure S2. DiB3/F74V and DiB3/F53L super-resolution performance in fixed HeLa cells. (A) Photostability in the localization microscopy setup of DiB3 mutants. The graph shows the normalized number of localizations per frame (Field of view was illuminated with $1.1 \mathrm{~kW} \mathrm{~cm}^{-2}$ of $488 \mathrm{~nm}$ laser light in case of DiB3/F74V imaging and $5.5 \mathrm{~kW} \mathrm{~cm}{ }^{-2}$ of $561 \mathrm{~nm}$ laser light in case of DiB3/F53L imaging). (B) The histogram of detected photons per single-molecule event; vertical lines represent median values of photons. 


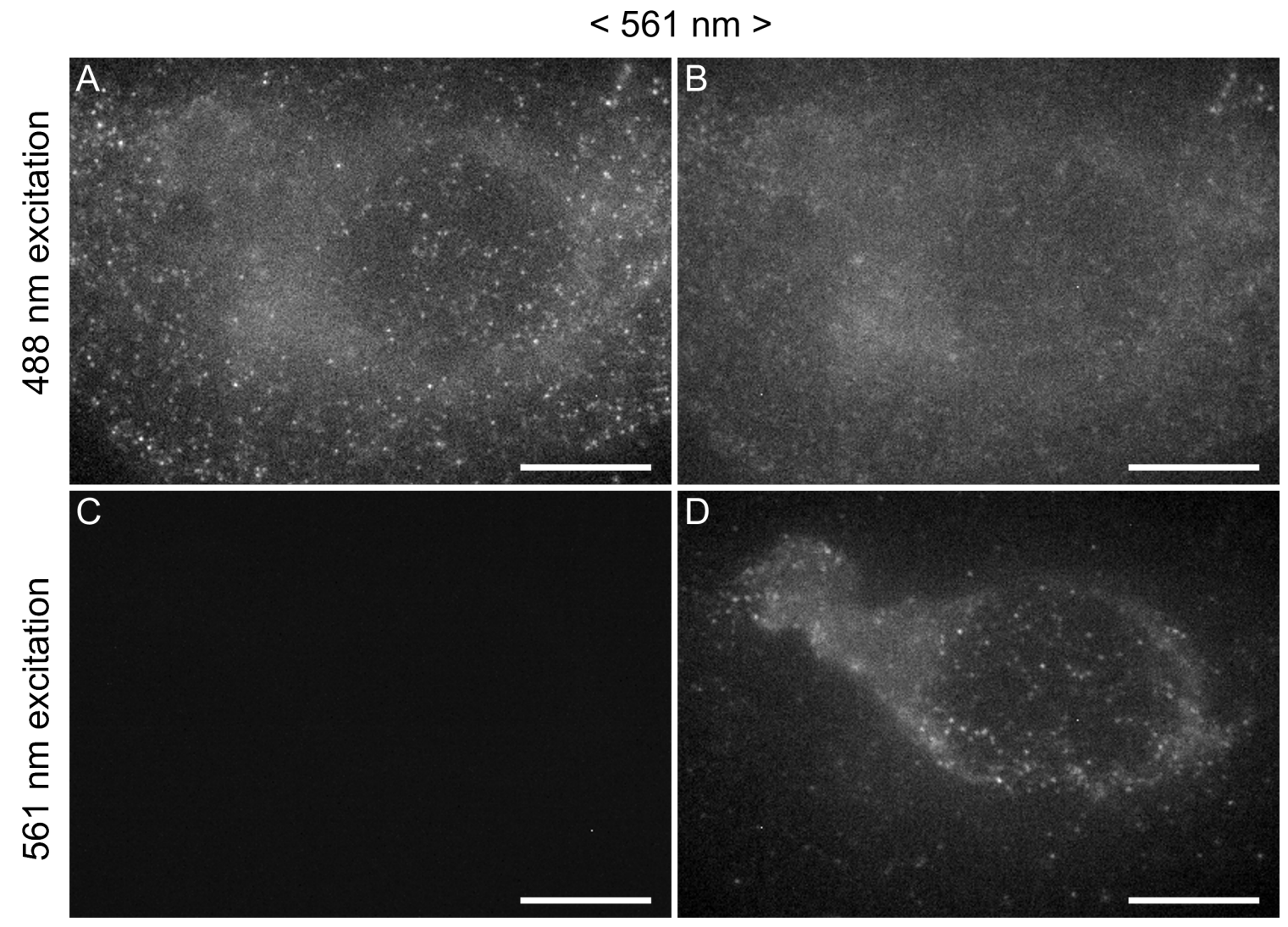

Figure S3. Single raw frames corresponding to the render in Figure 5. Imaging of vimentinDiB3/F53L and cytokeratin-DiB3/F74V in live HeLa cells transiently co-transfected with two constructs in the presence of $15 \mathrm{nM}$ M739. Individual frames of live HeLa cells with 488 excitation (A and B) or with $561 \mathrm{~nm}$ excitation (C and D). Emission is divided by a beamsplitter $(561 \mathrm{~nm})$ and detected simultaneously on two regions of the camera sensor. Panels A and C show detection at wavelengths $<561 \mathrm{~nm}$ and panels B and D - detection at wavelengths $>561 \mathrm{~nm}$. Scale bars are $10 \mu \mathrm{m}$. 


\section{Supplementary Movies}

Supplementary Movie 1. Movie showing 1000 frames of vimentin-DiB2 in live HeLa cells in the presence of 15nM M739. Plays at $30 \mathrm{fps}$ (acquisition speed $30 \mathrm{~Hz}$ ).

Supplementary Movie 2. Movie showing 1000 frames of vimentin-DiB3 in live HeLa cells in the presence of 15nM M739. Plays at $30 \mathrm{fps}$ (acquisition speed $30 \mathrm{~Hz}$ ).

Supplementary Movie 3. Movie showing 1000 frames of vimentin-DiB3/F74V in live HeLa cells in the presence of 15nM M739. Plays at $30 \mathrm{fps}$ (acquisition speed $30 \mathrm{~Hz}$ ).

Supplementary Movie 4. Movie showing 1000 frames of vimentin-DiB3/F53L/F74L/L129M in live HeLa cells in the presence of 15nM M739. Plays at $30 \mathrm{fps}$ (acquisition speed $100 \mathrm{~Hz}$ ).

Supplementary Movie 5. Movie showing 1000 frames of vimentin-DiB3/F53L in live HeLa cells in the presence of 15nM M739. Plays at $30 \mathrm{fps}$ (acquisition speed $30 \mathrm{~Hz}$ ).

Supplementary Movie 6. Movie showing 1000 frames of vimentin-DiB3/F74V in fixed HeLa cells in the presence of 15nM M739. Plays at $30 \mathrm{fps}$ (acquisition speed $100 \mathrm{~Hz}$ ).

Supplementary Movie 7. Movie showing 1000 frames of vimentin-DiB3/F53L in fixed HeLa cells in the presence of 15nM M739. Plays at $30 \mathrm{fps}$ (acquisition speed $30 \mathrm{~Hz}$ ).

Response movie 8. Movie showing 10 reconstructed frames of vimentin-DiB3/F53L in live HeLa cells in the presence of $15 \mathrm{nM} \mathrm{M739.} \mathrm{Each} \mathrm{frame} \mathrm{was} \mathrm{reconstructed} \mathrm{from} \mathrm{3,000} \mathrm{raw} \mathrm{frames,}$ 30,000 frames total. Plays at $10 \mathrm{fps}$ (acquisition speed $30 \mathrm{~Hz}$ ). Scale bar is $2 \mu \mathrm{m}$.

Response movie 9. Movie showing 10 reconstructed frames of vimentin-DiB3/F53L in live HeLa cells in the presence of $15 \mathrm{nM} \mathrm{M739.} \mathrm{Each} \mathrm{frame} \mathrm{was} \mathrm{reconstructed} \mathrm{from} \mathrm{3,000} \mathrm{raw} \mathrm{frames,}$ 30,000 frames total. Plays at $10 \mathrm{fps}$ (acquisition speed $60 \mathrm{~Hz}$ ). Scale bar is $2 \mu \mathrm{m}$. 


\section{Materials and methods}

Molecular cloning, overexpression, and purification of DiB1-3 and their mutants

The parental DiB1-3 were obtained as described in our earlier publication ${ }^{1}$. Site-directed mutagenesis of $\mathrm{DiB} 1$ and $\mathrm{DiB} 3$ was performed using the polymerase incomplete primer extension technique ${ }^{2}$. The DiB1-pBad and DiB3-pBad constructs were used as templates. The mutations (bold in underlined codon) were introduced using a pair of primers: DiB1/W139S: 5'-TAC CTGAGCATAAACTCCCGCACGCCAACCATTTCT-3' (forward coding strand) and 5'GTTTATGCTCAGGTAGTCGCGGTCCGGGC-3' (complementary strand); DiB1/V74F： 5'CTGAATTTTATTAATAAAGGCTATAACCCTGACAGAGGAATGTGGC-3' (forward coding strand) and 5'-ATTAATAAAATTCAGGCCG CCGTCATCA-3' (complementary strand); DiB3/F53L: 5'-CACCGCCTAGAACGTGGACTGGAAAAAGTCACCG-3' (forward coding strand) and 5'-ACGTTCTAGGCGGTGATCAAAACGGGCAATC-3' (complementary strand); DiB3/F74V: 5'-CTGAATGTAATTAATAAAGGCTATAACCCTGACAGAGGAATG TGGC-3' (forward coding strand) and 5'-ATTAATTACATTCAGGCCGCCGTCATC-3' (complementary strand); DiB3/F108L: 5'-GTGTCACTATTTGGTCCTTTCTATGGCGGT TATACG-3' (forward coding strand) and 5'-ACCAAATAGTGACACTTTCAGCGCAGCGCG-3' (complementary strand). The correctness of the resulting vectors was confirmed by sequencing. The mutated proteins were overexpressed and purified as described for the wild-type protein below. H2BDiB3/F53L and H2B-DiB3/F74V plasmids were constructed from H2B-DiB3 using AQUA cloning ${ }^{3}$. Dib3/F53L fusion with vimentin was performed following Golden Gate Assembly protocol $^{4-6}$.

DiB1-3 and their mutants were expressed in XJb (DE3) Autolysis (Zymo Research) E. coli strain. One bacterial colony was inoculated into $15 \mathrm{ml}$ of LB broth and grown overnight (16-18 h) at $30^{\circ} \mathrm{C}$. Inoculate was added to $1 \mathrm{~L}$ of $\mathrm{LB}$ broth and grown to $\mathrm{OD}_{600} 1$ at $37^{\circ} \mathrm{C}$. The temperature was then decreased to $18^{\circ} \mathrm{C}$, and $4 \mathrm{ml}$ of $20 \% \mathrm{~L}$-arabinose solution was added to induce protein expression. The culture was grown for $\sim 18$ hours. The cells were harvested and resuspended in $35 \mathrm{ml}$ of binding buffer (50 mM HEPES, $500 \mathrm{mM} \mathrm{NaCl}$, and $20 \mathrm{mM}$ imidazole, $\mathrm{pH}$ 7.6). The cells were disrupted by sonication using four bursts for a total duration of $4 \mathrm{~min}$, with $20 \mathrm{~s}$ intervals for cooling. Cell debris was pelleted by centrifugation at $25,000 \times \mathrm{g}$ for $30 \mathrm{~min}$ at $4^{\circ} \mathrm{C}$. The supernatant was applied to a column packed with $5 \mathrm{~mL}$ of HisTrap HP resin (GE Healthcare), 
connected to a VacMan manifold (Promega), and the chromatographic process was accelerated with a vacuum pump. After binding, the column was washed with $200 \mathrm{~mL}$ of the binding buffer, and the protein was eluted with $25 \mathrm{~mL}$ of elution buffer (50 mM HEPES pH 7.6, $500 \mathrm{mM} \mathrm{NaCl}$, $400 \mathrm{mM}$ imidazole). The flow-through was collected, concentrated to $3 \mathrm{~mL}$ and applied onto a HiLoad Superdex 200 16/60 column (GE Healthcare) equilibrated with $20 \mathrm{mM}$ TRIS-HCl pH 7.5, $200 \mathrm{mM} \mathrm{NaCl}$. Size exclusion chromatography yielded a homogeneous protein fraction.

\section{Complex preparation and crystallization}

The design of the Blc lipocalin fluorescent complex with fluorogen M739 has been described in detail in our previous publication ${ }^{1}$. To prepare 1:1 lipocalin complex with M739 $10 \mu 1$ of $0.1 \mathrm{M}$ DMSO stock solution of M739 was added to $1 \mathrm{ml}$ of $16 \mathrm{mg} / \mathrm{ml}$ protein solution in $20 \mathrm{mM}$ Tris $\mathrm{pH}$ 7.0, $200 \mathrm{mM} \mathrm{NaCl}$ and incubated at room temperature for 10 minutes. Crystals of DiB1 (Blc mutant complex with M739) were grown by hanging drop vapor diffusion method at $20^{\circ} \mathrm{C}$. Each drop consisted of $2 \mu \mathrm{l}$ of $\sim 16 \mathrm{mg} / \mathrm{ml}$ protein solution in $20 \mathrm{mM}$ Tris $\mathrm{pH}$ 7.0, $200 \mathrm{mM} \mathrm{NaCl}$, mixed with an equal amount of reservoir solution of $0.1 \mathrm{M} \mathrm{Na}$ citrate $\mathrm{pH}$ 5.0, 30\% PEG 550MME, 0.2M $\mathrm{NaSCN}$. The crystals reached their final size in two weeks.

\section{X-ray data collection, structure solution, and crystallographic refinement}

Diffraction data were collected from a single crystal flash-cooled in a $100 \mathrm{~K}$ nitrogen stream. Before cooling, the crystal was transferred to a cryo-protecting solution containing $20 \%$ glycerol and $80 \%$ of reservoir solution. The data were collected at a wavelength of $1 \AA$ with MAR300 CCD detector at SER-CAT beamline 22ID (Advanced Photon Source, Argonne National Laboratory, Argonne, IL) and were processed with $H K L 2000^{7}$.

The crystal structure of DiB1 was solved by the molecular replacement method with MOLREP ${ }^{8}$ using the coordinates of Blc monomer in apo-form (PDB ID: 1QWD ${ }^{9}$ ) as a search model. Structure refinement was performed with REFMAC ${ }^{10}$, alternating with the manual revision of the model, using COOT ${ }^{11}$. Crystallographic data and refinement statistics are given in Table 1. The coordinates and structure factors of DiB1 have been deposited in the Protein Data Bank under accession code $6 \mathrm{UBO}$.

Characterization of M739 complexes with DiB1-3 variants 
The absorbance and emission spectra were recorded on Duetta Fluorescence and Absorbance Spectrometer (Horiba) in $50 \mathrm{mM}$ Tris- $\mathrm{HCl}$ pH 7.5, $200 \mathrm{mM} \mathrm{NaCl}$ background buffer. All M739 aqueous solutions were prepared daily from $\mathrm{DMSO} \times 100$ stock and concentration of the aqueous stock solution was estimated from its absorbance spectra. The concentration of protein solutions was determined by its absorbance at $280 \mathrm{~nm}$ using extinction coefficients calculated by the ProtParam tool.

Lipocalin:M739 binding constants were estimated from fluorescent titration measurements following the approach described by Copeland et al. ${ }^{12}$, where increasing amounts of protein were added to the solution of M739 until the constant level of fluorescence was reached. We assumed 1:1 binding stoichiometry ${ }^{1}$ and fitted experimental data in a binding isotherm equation: $F=F_{0}+\frac{F_{\max }[P]}{\left(K_{D}+[P]\right)}$, where $F$ is the fluorescence intensity in the presence of a given concentration of the protein $[P], F_{0}$ is the fluorescence in the absence of the protein $[P]=0$, and $F_{\max }$ is the fluorescence when all fluorogen is bound.

Fluorescence enhancement of M739 was measured as following. We first excited $1.16 \mu \mathrm{M}$ M739 solution with $520 \mathrm{~nm}$ light, and recorded its emission spectrum, then added a 10-fold excess of lipocalin and recorded excitation spectra again, observing wavelength-independent fluorescence enhancement in the presence of FAP. To compensate for presence of the free M739 in solution, we calculated the fraction of the free $(F, \alpha)$ and bound fluorogen $(P F, 1-\alpha)$ and for each point of FAP-M739 emission spectra calculated the true fluorescence intensity that would have been achieved if all fluorogen were bound, $F_{P F}^{\lambda}(\operatorname{corr})$ :

$$
F_{P F}^{\lambda}(\operatorname{corr})=\frac{F_{m e a s}^{\lambda}-\alpha F_{M 739}^{\lambda}}{1-\alpha}
$$

where $F_{M 739}^{\lambda}$ and $F_{\text {meas }}^{\lambda}$ are fluorescence intensities at a given wavelength in solution of M739 before and after addition of FAP, respectively. $F_{P F}^{\lambda}(\operatorname{corr})$ was then compared with $F_{M 739}^{\lambda}$ to estimate the fluorescence enhancement achieved by each particular FAP. The extinction coefficient and quantum yield of M739 complexes with lipocalins was determined in the presence of the protein excess to ensure a complete at least 90\% fluorogen binding. Quantum yields were determined by direct comparison with purified fluorescent protein Venus (QY $0.57, \lambda_{\text {ex }} / \lambda_{\text {em }}$ 
$515 / 528 \mathrm{~nm})$. The obtained spectra were corrected to separate contributions of free and bound fluorogen in both absorbance and emission spectra.

\section{Evaluation of photostability of DiB3/F74V and DiB3/F53L variants}

Confocal microscopy and photobleaching experiments were performed with an inverted Leica microscope DMIRE2 TCS SP2 (Leica, Wetzlar, Germany) equipped with HCX PL APO lbd.BL $63.0 \times 1.40$ OIL objective, excitation by $543 \mathrm{~nm}$ laser line $(26 \mu \mathrm{W})$, and $488 \mathrm{~nm}$ laser line $(32$ $\mu \mathrm{W}) .890 \mu \mathrm{m}^{2}$ region was scanned with a $32 \mu \mathrm{W} 488 \mathrm{~nm}$ laser in case of bleaching EGFP, DiB2, and DiB3/ F74V, and DiB3/F53L/F74L/L129M. $221 \mu \mathrm{m}^{2}$ regions were scanned with a $26 \mu \mathrm{W}$ $543 \mathrm{~nm}$ laser in case of bleaching mKate2 and DiB3/F53L. Before imaging, cells were placed in the HHBS media (Hanks' Buffer with 20 mM HEPES).

\section{Protein-PAINT super-resolution imaging}

Single-molecule localization super-resolution imaging of living cells was carried out on Nanoimager S (Oxford Nanoimaging). The evaluation of single-molecule properties of lipocalin mutants (Fig. 4, Supplementary Fig. S3) was performed with $45 \mathrm{~mW}$ (488 nm) and $216 \mathrm{~mW}$ (561 $\mathrm{nm}$ ) laser light intensities. Typical acquisitions were 5,000 frames taken at a frequency of $30 \mathrm{~Hz}$. Prolonged super-resolution imaging of DiB3/F53L was performed with 30 and $60 \mathrm{~Hz}$ acquisition frequencies, 30,000 frames were taken with $1.4 \mathrm{~kW} \mathrm{~cm}^{-2}$ of $561 \mathrm{~nm}$ laser light. Two-color superresolution imaging was performed under the following imaging conditions: 10,000 frames, acquisition frequency $30 \mathrm{~Hz}$, imaging cycle: 100 frames with $1.8 \mathrm{~kW} \mathrm{~cm}{ }^{-2} 488 \mathrm{~nm}$ laser, 100 frames with $1.3 \mathrm{~kW} \mathrm{~cm}^{-2}$ of $561 \mathrm{~nm}$ laser. 


\section{Supplementary References}

1. Bozhanova, N. G.; Baranov, M. S.; Klementieva, N. V.; Gavrikov, A. S.; Sarkisyan, K. S.; Yampolskiy, I. V.; Zagaynova, E. V.; Lukyanov, S. A.; Lukyanov, K. A.; Mishin, A. S., Protein labeling for live cell fluorescence microscopy with a highly photostable renewable signal. Chem Sci 2017, 8 (10), 7138-7142.

2. Klock, H. E.; Lesley, S. A., The Polymerase Incomplete Primer Extension (PIPE) method applied to high-throughput cloning and site-directed mutagenesis. Methods in molecular biology (Clifton, N.J.) 2009, 498, 91-103.

3. Beyer, H. M.; Gonschorek, P.; Samodelov, S. L.; Meier, M.; Weber, W.; Zurbriggen, M. D., AQUA Cloning: A Versatile and Simple Enzyme-Free Cloning Approach. PLoS One 2015, 10 (9), e0137652.

4. $\quad$ Engler, C.; Gruetzner, R.; Kandzia, R.; Marillonnet, S., Golden gate shuffling: a one-pot DNA shuffling method based on type IIs restriction enzymes. PLoS One 2009, 4 (5), e5553.

5. Engler, C.; Kandzia, R.; Marillonnet, S., A one pot, one step, precision cloning method with high throughput capability. PLoS One 2008, 3 (11), e3647.

6. Engler, C.; Marillonnet, S., Generation of families of construct variants using golden gate shuffling. Methods in molecular biology (Clifton, N.J.) 2011, 729, 167-81.

7. Minor, W.; Cymborowski, M.; Otwinowski, Z.; Chruszcz, M., HKL-3000: the integration of data reduction and structure solution--from diffraction images to an initial model in minutes. Acta Crystallogr., Sect. D: Biol. Crystallogr. 2006, 62 (Pt 8), 859-66.

8. Vagin, A.; Teplyakov, A., Molecular replacement with MOLREP. Acta Crystallogr., Sect. D: Biol. Crystallogr. 2010, 66 (Pt 1), 22-5.

9. Campanacci, V.; Nurizzo, D.; Spinelli, S.; Valencia, C.; Tegoni, M.; Cambillau, C., The crystal structure of the Escherichia coli lipocalin Blc suggests a possible role in phospholipid binding. FEBS letters 2004, 562 (1-3), 183-8.

10. Murshudov, G. N.; Skubak, P.; Lebedev, A. A.; Pannu, N. S.; Steiner, R. A.; Nicholls, R. A.; Winn, M. D.; Long, F.; Vagin, A. A., REFMAC5 for the refinement of macromolecular crystal structures. Acta Crystallogr., Sect. D: Biol. Crystallogr. 2011, 67 (Pt 4), 355-67.

11. Emsley, P.; Lohkamp, B.; Scott, W. G.; Cowtan, K., Features and development of Coot. Acta Crystallogr., Sect. D: Biol. Crystallogr. 2010, 66 (Pt 4), 486-501.

12. Copeland, R. A., Enzymes : a practical introduction to structure, mechanism, and data analysis. 2nd ed.; Wiley: New York, 2000; p xvi, 397 p. 